ISSN 0103-8478

\title{
Scarabaeoidea (Insecta: Coleoptera) no Cerrado brasileiro: estado atual do conhecimento
}

\author{
Scarabaeoidea (Insecta: Coleoptera) in the Brazilian Cerrado: current state of knowledge
}

\author{
Milena Luçardo ${ }^{\mathrm{I}}$ Charles Martins de Oliveira ${ }^{\mathrm{II}}$ Marina Regina Frizzas ${ }^{\mathrm{III}}$
}

\section{- REVISÃO BIBLIOGRÁFICA -}

RESUMO

Besouros pertencentes à superfamília Scarabaeoidea ocupam habitats variados, possuem hábitos alimentares diversificados, desempenham importante papel ecológico e diversas espécies apresentam importância agrícola. No entanto, estudos com esse grupo na região do Cerrado são escassos. Nesta revisão realizou-se um levantamento dos artigos publicados nos últimos 30 anos a respeito dos Scarabaeoidea no Cerrado. Foram recuperados 64 artigos, realizados em nove unidades da federação, que focavam quatro temas principais: espécies praga, aspectos bioecológicos, biodiversidade e importância ecológica, e técnicas e metodologias de coleta de Scarabaeoidea. Os resultados desta revisão indicam que poucos estudos foram realizados com os Scarabaeoidea no Cerrado brasileiro nas últimas décadas frente à importância e diversidade desse grupo de insetos.

Palavras-chave: Scarabaeidae, Melolonthidae, biodiversidade, bioecologia, besouros.

\section{ABSTRACT}

Beetles belonging to the superfamily Scarabaeoidea occupy different habitats, present feeding habits diversified, play an important ecological role and several species have agricultural importance. However, studies with this group in the Brazilian Cerrado are scarce. In this review we carried out a survey of scientific articles published in the past 30 years concerning Scarabaeoidea in the Cerrado. Were found 64 studies in nine Brazilian states. The studies focused on four main topics: pest species, bioecology, biodiversity and ecological importance, techniques and methodologies for collecting Scarabaeoidea. The results of this review indicate that few studies have been conducted with Scarabaeoidea in the Cerrado in recent decades compared to the importance and diversity of this group of insects.

Key words: Scarabaeidae, Melolonthidae, biodiversity, bioecology, beetles.

\section{INTRODUÇÃO}

Não existe consenso quanto à divisão de famílias e subfamílias dentro dos Scarabaeoidea e o número relativo a esses táxons varia de acordo com o sistema de classificação adotado. Adotou-se nesta revisão a divisão proposta por MORÓN (2010) que inclui 12 famílias (Cetoniidae, Geotrupidae, Glaphyridae, Glaresidae, Hybosoridae, Lucanidae, Melolonthidae, Ochodaeidae, Passalidae, Pleocomidae, Scarabaeidae e Trogidae).

Os Scarabaeoidea são cosmopolitas, ocupando quase todos os ambientes do planeta. Desempenham diversas funções ecológicas nos ecossistemas provendo serviços variados e auxiliando na manutenção desses ambientes (NICHOLS et al., 2008). Alguns Scarabaeoidea são considerados pragas de diversas espécies de plantas cultivadas, especialmente em sua fase larval, podendo atingir elevada importância econômica devido às perdas que podem proporcionar (ARAGÓN et al., 2001).

O Cerrado, apesar de conter cerca de 30\% da biodiversidade brasileira (DIAS, 1992), encontrase na lista dos biomas de alta biodiversidade mais ameaçados do mundo (MITTERMEIER et al., 2005). O conhecimento a cerca dos organismos que compõem o Cerrado e suas inter-relações torna-se essencial para as políticas de conservação e de destinação do uso da terra. Os Scarabaeoidea formam um elo importante

ICentro Universitário de Brasília (UniCEUB), Faculdade de Ciências da Saúde (FACS), Asa Norte, Brasília, DF, Brasil.

IIEmbrapa Cerrados, Rod. BR 020, km 18, 73310-970, CP 08223, Planaltina, DF, Brasil. E-mail:charles.oliveira@embrapa.br. Autor para correspondência.

IIIDepartamento de Zoologia, Instituto de Ciências Biológicas, Universidade de Brasília (UnB), Brasília, DF, Brasil. 
dentro dos ecossistemas naturais e antropizados e desempenham um papel importante para o equilíbrio do bioma Cerrado.

Esta revisão objetivou reunir o conhecimento a respeito dos Scarabaeoidea na área de abrangência do Cerrado por meio dos estudos científicos desenvolvidos com esta superfamília nos últimos 30 anos. A seleção dos estudos foi realizada com base na data de publicação (1982 a 2012) e foram utilizados artigos publicados em revistas indexadas com corpo editorial, restritos ao bioma Cerrado. As informações contidas neste estudo foram obtidas por meio de buscas realizadas nas bases Scielo, Portal de Periódicos da CAPES, Google Acadêmico, BioOne Online Journals, CAB Abstracts, Web of Knowlodge e Jstor. As palavraschave, e suas combinações, utilizadas para as buscas bibliográficas foram Scarabaeoidea, Brazilian Savannah, Cerrado, Cetoniidae, Geotrupidae, Glaphyridae, Glaresidae, Hybosoridae, Lucanidae, Melolonthidae, Ochodaeidae, Passalidae, Pleocomidae, Scarabaeidae e Trogidae. Foram utilizadas também as publicações citadas nos artigos obtidos na busca, mas que não foram recuperadas por meio das palavras-chave.

No total foram encontrados 64 trabalhos, sendo 14 sobre espécies consideradas pragas, 15 abordavam aspectos bioecológicos, 41 trabalhos relacionados à biodiversidade e importância ecológica e 35 continham informações sobre técnicas e metodologias de coleta. Os estudos estão distribuídos em nove estados brasileiros: Mato Grosso do Sul (30), Minas Gerais (19), Goiás (12), Distrito Federal (7), Mato Grosso (4), Maranhão (2), Piauí (2), Paraná (1) e Tocantins (1).

\section{Estudos sobre espécies praga}

Foram encontradas 15 espécies de Scarabaeoidea consideradas pragas, distribuídas em três estados brasileiros. Todas as espécies identificadas como pragas no Cerrado brasileiro pertencem à família Melolonthidae. As larvas dessas espécies, denominadas comumente de corós, atacam o sistema radicular, interferem no crescimento e/ou ocasionam a morte das plantas, podendo reduzir drasticamente o estande e a produtividade das culturas atacadas quando em altas populações (OLIVEIRA et al., 2007; OLIVEIRA et al., 2008; COUTINHO et al., 2011; RODRIGUES et al., 2011). Os adultos de algumas espécies também podem causar prejuízos (OLIVEIRA et al., 2008; OLIVEIRA \& ÁVILA, 2011).

De forma geral os prejuízos causados por Scarabaeoidea pragas no Cerrado se concentram no período chuvoso havendo um sincronismo de ocorrência da fase larval com os plantios de verão e são mais acentuados quando as plantas são atacadas na fase inicial de desenvolvimento (OLIVEIRA et al., 2007; OLIVEIRA et al., 2008).

Culturas atacadas

A polifagia tem sido apontada como uma das características de algumas espécies de Scarabaeoidea consideradas praga no Cerrado brasileiro (RODRIGUES et al., 2011). Larvas de Aegopsis bolboceridus (Thomson) foram observadas causando $100 \%$ de perdas em hortaliças e feijão no Distrito Federal (OLIVEIRA et al., 2008). Na cultura da soja registram-se as espécies Phyllophaga capillata (Blanchard) no Distrito Federal (OLIVEIRA et al., 2007), Liogenys fuscus Blanchard em Goiás (COSTA et al., 2009) e Mato Grosso do Sul (RODRIGUES et al., 2011), Cyclocephala forsteri Endrodi, Anomala testaceipennis Blanchard e Phyllophaga cuyabana Moser no Mato Grosso do Sul (SANTOS \& ÁVILA, 2007). Na cultura do milho são encontradas causando danos as espécies A. testaceipennis, Liogenys suturalis Blanchard, L. fuscus e P. cuyabana no Mato Grosso do Sul (SANTOS \& ÁVILA, 2009a; RODRIGUES et al., 2011) e A. bolboceridus em Goiás (OLIVEIRA et al., 2008). Em cana-de-açúcar são registradas as espécies L. fuscus, Anomonyx sp., C. forsteri e C. verticalis Burmeister (COUTINHO et al., 2011). Em áreas de pastagens as espécies $\boldsymbol{C}$. verticalis e $\boldsymbol{A}$. testaceipennis foram registradas em plantas do gênero Brachiaria (RODRIGUES et al., 2008a; RODRIGUES et al., 2010a). Em macaúba observou-se, associado ao sistema radicular, as espécies Astaena sp., C. verticalis, Leucothyreus dorsalis Blanchard, Leucothyreus sp., L. fuscus e Phyllophaga sp. (PUKER et al., 2009; OLIVEIRA \& ÁVILA, 2011). Uma espécie não identificada de Melolonthidae foi observada causando danos em trigo no Mato Grosso do Sul (ÁVILA \& PÍPOLO, 1992). Com relação às formas adultas registrou-se $\boldsymbol{C}$. forsteri atacando a inflorescência de plantas de macaúba e Leucothyreus albopilosus Ohaus causando lesões em troncos de eucalipto (PUKER et al., 2011b).

Manejo de espécies pragas

Os estudos realizados no Cerrado têm demonstrado que o manejo desse grupo de pragas depende da adoção conjunta de medidas que englobam o controle químico, cultural e biológico. O controle químico por meio da utilização do tratamento inseticida de sementes ou da pulverização de inseticidas no sulco de plantio tem sido utilizado para o manejo de corós 
rizófagos. Estudos de campo indicaram que a aplicação de inseticidas dos grupos organofosforados, pirazóis e neonicotinóides no sulco de plantio e/ou em tratamento de semente foi eficiente no controle de $\boldsymbol{L}$. fuscus em milho (SANTOS et al., 2008). Quanto ao controle biológico existe apenas o registro de parasitismo de larvas de $\boldsymbol{L}$. suturalis pelo taquinídeo Ptilodexia Brauer \& Bergenstamm (SANTOS \& ÁVILA, 2009b).

Estudos sobre aspectos bioecológicos

Dos estudos relacionados com aspectos bioecológicos de Scarabaeoidea, 9 tratavam de espécies praga. Com exceção de $\boldsymbol{A}$. testaceipennis e Digitonthophagus gazella Fabricius que se reproduzem várias vezes ao ano (MARTINS \& CONTEL, 1997; FONSECA\& KERR, 2005; RODRIGUES et al., 2008a), as espécies de Sacarabaeoidea que ocorrem no Cerrado apresentam apenas uma geração por ano (RODRIGUES et al., 2012). A duração do ciclo biológico variou, sendo o ciclo mais curto de $\boldsymbol{D}$. gazella com cerca de um mês (FONSECA \& KERR, 2005) e os mais longos variando de 10 a 12 meses como para Pelidnota fulva Blanchard (RODRIGUES \& FALCO, 2011).

\section{Ciclo biológico}

Os ovos, de modo geral, apresentam coloração branca, inicialmente são elípticos e posteriormente assumem um formato esférico (RODRIGUES et al., 2008a; RODRIGUES et al., 2010b; PUKER et al., 2011a; RODRIGUES et al., 2012). O período embrionário pode chegar a 22,8 dias (SANTOS \& ÁVILA, 2009a). Na maioria das espécies as larvas podem ser encontradas no solo entre os meses de novembro e março (RODRIGUES et al., 2008a; RODRIGUES et al., 2008b; PUKER et al., 2009; SANTOS \& ÁVILA, 2009a; COUTINHO et al., 2011), com algumas exceções como $\boldsymbol{L}$. fuscus e $\boldsymbol{C}$. forsteri onde a fase larval ocorre em maior densidade entre abril e setembro (COUTINHO et al., 2011). O período larval pode durar até 269 dias como em $\boldsymbol{P}$. fulva (RODRIGUES \& FALCO, 2011). As pupas possuem coloração variando de branco-amarelado a marromcaramelo e, em algumas espécies, pode-se notar o dimorfismo sexual já neste estágio (RODRIGUES et al., 2010b).

\section{Comportamento}

$\mathrm{Na}$ fase adulta as revoadas geralmente iniciam-se com as primeiras chuvas, entre os meses de agosto/setembro, estendendo-se até dezembro. Essas espécies sincronizam sua reprodução com a época de plantio das culturas das quais se alimentam (RODRIGUES et al., 2008a; RODRIGUES et al., 2008b; PUKER et al., 2009; SANTOS \& ÁVILA, 2009a; COUTINHO et al., 2011). Durante este período, os adultos emergem do solo, preferencialmente ao anoitecer, para procurar um parceiro para o acasalamento. O início da atividade de voo ocorre entre 18 e 19h (RODRIGUES et al., 2010a) e termina entre 01 e 02h para algumas espécies (SANTOS \& ÁVILA, 2009a) e para outras às 05h30 (RODRIGUES et al., 2010b). Fêmeas e machos se encontram, geralmente por intermédio de feromônios, e depois da cópula as fêmeas retornam ao solo para depositar os ovos (SANTOS \& ÁVILA, 2007; VAZ-DE-MELLO \& GÉNIER, 2009; RODRIGUES et al., 2010b). No caso de espécies coprófagas, os ovos são depositados em esferas de fezes bovinas (RODRIGUES \& FLECHTMANN, 1997; FONSECA \& KERR, 2005).

Estudos sobre biodiversidade e importância ecológica Aspectos relacionados à biodiversidade e à importância ecológica de Scarabaeoidea se destacaram, representando $64 \%$ de todos os estudos realizados no Cerrado.

\section{Descrição de novas espécies}

Diversas espécies foram descritas no bioma Cerrado. De espécimes coletados no Mato Grosso do Sul foi descrito o gênero Flechtmannia Stebnicka e as espécies Selviria matogrossoensis Stebnicka e Lomanoxoides selviriaensis Stebnicka (STEBNICKA, 1999), além deAnomiopus birai Canhedo de espécimes de Mato Grosso e Mato Grosso do Sul (CANHEDO, 2006). Para Minas Gerais, foram descritas $S$. anneae Stebnicka (STEBNICKA, 2005), Daimothoracodes confossus Ocampo e Vaz-de-Mello (OCAMPO \& VAZ-DE-MELLO, 2002), Pedaridium louzadaorum Vaz-de-Mello \& Canhedo e $\boldsymbol{P}$. zanunciorum Vaz-deMello \& Canhedo (VAZ-DE-MELLO \& CANHEDO, 1998), A. serranus Canhedo e A. pumilius Canhedo (CANHEDO, 2006) e Ontherus (Ontherus) gladiator Genier (GENIER, 1998). Para o Distrito Federal, registraram-se como novas espécies $\boldsymbol{D}$. rugomarginatus Ocampo (OCAMPO, 2005) e A. sulcatus Canhedo (CANHEDO, 2006). A espécie Neoathyreus moraguesi Howden foi descrita com base em um espécime coletado no estado de Goiás (HOWDEN, 2006) e A. mourai Canhedo descrita de espécimes coletados em Tocantins (CANHEDO, 2006).

Registros de ocorrência

No Mato Grosso do Sul registrou-se a presença de Bolbites onitoides Harold, sendo este o primeiro registro do gênero no Brasil (VAZ-DE-MELLO \& GROSSI, 2010). No Cerrado maranhense houve 
registro da espécie Brachysiderus (Minisiderus) mielkeorum Grossi e Grossi (GROSSI \& GROSSI, 2005) e novos registros para o estado do Piauí de Coprophanaeus (Coprophanaeus) cyanescens (Olsoufieff) e C. (Metallophanaeus) pertyi (GILLETT et al., 2010). As espécies Dendropaemon sp., Diabrotics mimas L., Phanaeus (Notiophanaeus) malibaeus Blanchard e $\boldsymbol{C}$. (Coprophanaeus) acrisius (Macleay) estão associadas a áreas de Cerrado (GILLETT et al., 2010).

Revisões dos gêneros registraram sete espécies de Anomiopus Westwood e cinco de Chalepides Casey em áreas de Cerrado em diversos estados brasileiros (JOLY \& ESCALONA, 2002; CANHEDO, 2006). A espécie Iarupea attenuata (Harold) foi registrada em Minas Gerais e $\boldsymbol{L}$. tesari (Balthasar) em Mato Grosso e Minas Gerais (STEBNICKA, 1999).

Diversidade de espécies, importância e interações ecológicas

Espécies coprófagas de Scarabaeoidea, em sistemas agropastoris, desempenham papel relevante no controle biológico de parasitas que utilizam as fezes bovinas para depositar seus ovos, desestruturando a massa fecal e inviabilizando a oviposição do parasita (MARCHIORI et al., 2000b; MARCHIORI, 2003; MARCHIORI et al., 2003; KOLLER et al., 2007; BORGES et al., 2011; ABOT et al., 2012). Além das espécies coprófagas, poucos estudos têm sido realizados com relação à diversidade de espécies nos demais Scarabaeoidea.

Assim como os besouros coprófagos, espécies como P. fulva (RODRIGUES \& FALCO, 2011) e Astaena sp. (BRANCO et al., 2010) também proporcionam a ciclagem de nutrientes, porém alimentam-se de matéria vegetal em decomposição, auxiliando na incorporação da matéria orgânica no solo. Diversas espécies são atraídas por carcaças, utilizando-as como fonte de alimento (MARCHIORI et al., 2000a; ROSA et al., 2011).

Alguns Scarabaeoidea (Dynastis sp. e Strategus sp.) fazem parte da dieta de mamíferos, como canídeos, em momentos de pouca disponibilidade de alimento (UCHOA \& MOURA-BRITTO, 2004). Outras espécies apresentam potencial como polinizadores. As espécies Cyclocephala atricapilla Mannerheim, C. latericia Hohne e $\boldsymbol{C}$. octopunctata Burmeister foram observadas visitando flores de araticum e devido a abundância $\boldsymbol{C}$. octopunctata foi indicada como possível polinizador dessa espécie vegetal (CAVALCANTE et al., 2009).

Associações ecológicas com outras espécies foram encontradas. Adultos de L. albopilosus foram observados em cavidades de própolis de colmeias de Apis mellifera L. (PUKER et al., 2011b). A análise da flora intestinal de besouros da família Scarabaeidae apontou interações com microrganismos, atuando como prováveis dispersores de bactérias e fungos de importância ecológica, agrícola ou médica (NUNES \& FRIZZAS, 2007). Duas espécies de Dendropaemon e Tetramereia convexa (Harold) foram observadas em ninhos de Atta Fabricius, sugerindo relação ecológica com formigas cortadeiras (VAZ-DE-MELLO \& GÉNIER, 2009). Comportamento predatório foi registrado para Canthon virens (Mannerheim). Esta espécie foi observada predando formigas cortadeiras [Atta laevigata (Smith)] (SILVEIRA et al., 2006).

Em geral, mudanças na paisagem, desmatamento ou mesmo reflorestamentos alteram a estrutura da comunidade de escarabeídeos e a composição de espécies locais (GRIES et al., 2012), com raras exceções (RESENDE, 2012). Como são sensíveis às perturbações, estudos sugerem a utilização desses insetos como bioindicadores (ALMEIDA \& LOUZADA, 2009; BORGES et al., 2011; GRIES et al., 2012). As assembleias de espécies presentes em áreas abertas diferem das encontradas em ambientes de Cerrado mais fechado, possuindo algumas espécies restritas a cada tipo de área (ALMEIDA \& LOUZADA, 2009; NUNES et al., 2012), essas diferenças também podem ser observadas entre áreas de floresta e Cerrado (DURÃES et al., 2005) e áreas de reflorestamento, pastagens e vegetação natural de Cerrado (GRIES et al., 2012). Nas fitofisionomias de Cerrado, o campo sujo é o que apresenta maior diversidade de escarabeídeos (MILHOMEM et al., 2003).

Estudos sobre técnicas e metodologias de coleta

Tratando-se de Scarabaeoidea, existem diversas técnicas de amostragem que podem ser utilizadas. Os estudos realizados apontaram a utilização de armadilha luminosa, armadilha de queda (pitfall), armadilha de interceptação, coletas manuais, funil de Berlesse e, também, observações em laboratório e campo, sendo que alguns estudos utilizaram mais de uma técnica ou metodologia de coleta.

A armadilha luminosa tem sido amplamente utilizada para amostrar Scarabaeoidea noturnos fototrópicos positivos, mostrando eficiência na captura deste grupo (FLECHTMANN et al., 1995a; FLECHTMANN et al., 1995b; FLECHTMANN et al., 1995c; NUNES \& FRIZZAS, 2007; BRANCO et al., 2010; ABOT et al., 2012), principalmente para estudos de dinâmica populacional (RODRIGUES et al., 2008a; RODRIGUES et al., 2008b; COSTA et al., 
2009; SANTOS \& ÁVILA, 2009a; RODRIGUES et al., 2010a; RODRIGUES et al., 2010b; RODRIGUES \& FALCO, 2011). A armadilha de queda (pitfall) pode ser utilizada sem isca (SILVA et al., 2010) ou pode conter iscas que atraem uma gama mais específica de besouros denominados copronecrófagos. Os tipos de iscas mais utilizados foram fezes humanas (ALMEIDA et al., 2011; BORGES et al., 2011), fezes bovinas (AIDAR et al., 2000; KOLLER et al., 1999; KOLLER et al., 2007), fezes equinas (LOUZADA \& SILVA, 2009), pedaços de carcaças de animais em decomposição, como baço (ALMEIDA \& LOUZADA, 2009), fígado e carne bovina (MILHOMEM et al., 2003) e partes de frango (GILLETT et al., 2010), ou frutas (NUNES et al., 2012). Armadilhas de interceptação do tipo janela e Malaise (VAZ-DE-MELLO \& GÉNIER, 2009) não têm se mostrado efetivas para Scarabaeoidea em comparação a outras armadilhas (AUAD \& CARVALHO, 2011).

Amostragens manuais são realizadas tanto para larvas edáficas quanto adultos. Aliado a essas coletas, podem ser observados comportamentos reprodutivos (FONSECA \& KERR, 2005; SILVEIRA et al., 2006), de forrageamento (PUKER et al., 2009; OLIVEIRA \& ÁVILA, 2011) e interações ecológicas (PUKER et al., 2011b). O substrato onde se encontram pode ser coletado e depois quantificado o número de espécies (COUTINHO et al., 2011; RODRIGUES et al., 2011).

\section{CONCLUSÃO}

Os estudos sobre a superfamília Scarabaeoidea no Cerrado são restritos a poucas famílias, sendo Scarabaeidae e Melolonthidae as mais representativas. O número de trabalhos que envolvem espécies que não se encaixem nos hábitos copronecrófagos e herbívoros é bastante limitado. A maioria dos trabalhos aborda aspectos do comportamento e biologia de espécies considerada pragas. Mesmo para as famílias mais estudadas o número de trabalhos existente para o Cerrado brasileiro é insignificante frente ao número de espécies que habitam esse bioma. Estudos com Scarabaeoidea são de grande relevância, pois podem nortear a estruturação de políticas públicas visando a adoção de medidas de conservação para o grupo e de medidas de controle para o manejo integrado daquelas consideradas pragas.

\section{REFERÊNCIAS}

ABOT, A.R. et al. Abundance and diversity of coprophagous beetles (Coleoptera: Scarabaeidae) caught with a light trap in a pasture area of the Brazilian Cerrado. Studies on Neotropical Fauna and Environment, v.47, n.1, p.53-60, 2012. Disponível em: <http:// www.tandfonline.com/doi/abs/10.1080/01650521.2012. 662846>. Acesso em: 21 nov. 2012.

AIDAR, T. et al. Besouros coprófagos (Coleoptera: Scarabaeidae) coletados em aquidauana, MS, Brasil. Anais da Sociedade Entomológica do Brasil, v.29, n.4, p.817-820, 2000. Disponível em: <http://www.scielo.br/pdf/\%0D/aseb/v29n4/v29n4a23.pdf>. Acesso em: 21 out. 2012.

ALMEIDA, S. et al. Subtle land-use change and tropical biodiversity: dung beetle communities in Cerrado grasslands and exotic pastures. Biotropica, v.43, n.6, p.704-710, 2011. Disponível em: <http://onlinelibrary.wiley.com/doi/10.1111/ j.1744-7429.2011.00751.x/full>. Acesso em: 17 set. 2012. doi: 10.1111/j.1744-7429.2011.00751.x.

ALMEIDA, S.S.P.; LOUZADA, J.N.C. Estrutura da comunidade de Scarabaeinae (Scarabaeidae: Coleoptera) em fitofisionomias do Cerrado e sua importância para a conservação. Neotropical Entomology, v.38, n.1, p.32-43, 2009. Disponível em: <http:// www.scielo.br/pdf/ne/v38n1/03.pdf>. Acesso em: 01 out. 2012.

ARAGÓN, A. et al. Fauna de Coleoptera Melolonthidae en el rancho "La Joya", Atlixco, Puebla, México. Acta Zoológica Mexicana, v.83, p.143-164, 2001. Disponível em: <http://redalyc. uaemex.mx/pdf/575/57508308.pdf>. Acesso em: 01 out. 2012.

AUAD, A.M.; CARVALHO, C.A. de. Análise faunística de coleópteros em sistema silvipastoril. Ciência Florestal, v.21, n.1, p.31-39, 2011. Disponível em: <http://cascavel.cpd. ufsm.br/revistas/ojs-2.2.2/index.php/cienciaflorestal/article/ download/2745/1671>. Acesso em: 01 out. 2012.

ÁVILA, C.J.; PÍPOLO, A.E. Ocorrência e danos do "coró" (Coleoptera: Scarabaeidae - Melolonthinae) em trigo na região de Dourados, MS. Anais da Sociedade Entomologica do Brasil, v.21, n.1, p.261, 1992.

BORGES, N.F.J. et al. Scarabaeidae (Coleoptera) coprófagos em fragmentos de vegetação nativa (Cerrado) no perímetro urbano de Catalão, Goiás (Brasil). Acta Biológica Paranaense, v.40, n.3-4, p.73-85, 2011. Disponível em: <http://ojs.c3sl.ufpr.br/ojs-2.2.4/ index.php/acta/article/view/25129/>. Acesso em: 07 ago. 2012.

BRANCO, R.T.P.C. et al. Análise faunística de insetos associados à cultura da cana-de-açúcar, em área de transição floresta amazônica - cerrado (mata de cocal), no município de União - Piauí Brasil. Semina: Ciências Agrárias, v.31, supl.1, p.1113-1120, 2010. Disponível em: <http://www.uel.br/revistas/uel/index.php/ semagrarias/article/view/7961/6936>. Acesso em: 15 ago. 2012

CANHEDO, V.L. Revisão taxonômica do gênero Anomiopus Westwood, 1842 (Coleoptera, Scarabaeidae, Scarabaeinae). Arquivos de Zoologia, v.37, n.4, p.349-502, 2006. Disponível em: <http://www.revistasusp.sibi.usp.br/pdf/azmz/v37n4/a01v37n4. pdf $>$. Acesso em: 07 ago. 2012.

CAVALCANTE, T.R.M. et al. Polinização e formação de frutos em araticum. Bragantia, v.68, n.1, p.13-21, 2009. Disponível em: $<$ http://www.scielo.br/pdf/brag/v68n1/ a02v68n1.pdf >. Acesso em: 07 ago. 2012.

COSTA, R.B. et al. Captura de adultos de Liogenys fuscus (Coleoptera: Melolonthidae) com armadilha luminosa em área sob sistema de plantio direto. Bioscience Journal, v.25, n.3, p.1-8, 2009. Disponível em: <http://www.seer.ufu.br/index.php/ 
biosciencejournal/article/view/6900/4568>. Acesso em: 15 ago. 2012.

COUTINHO, G.V. et al. Bionomic data and larval density of Scarabaeidae (Pleurosticti) in sugarcane in the central region of Mato Grosso do Sul, Brazil. Revista Brasileira de Entomologia, v.55, n.3, p.389-395, 2011. Disponível em: <http://www.scielo.br/ pdf/rbent/v55n3/aop4211.pdf>. Acesso em: 02 dez. 2012.

DIAS, B.F.S. Cerrados: uma caracterização. In: DIAS, B.F.S. Alternativas de desenvolvimento dos Cerrados: manejo e conservação dos recursos naturais renováveis. Brasília: Funatura, 1992. p.11-25.

DURÃES, R. et al. Dung beetle (Coleoptera: Scarabaeidae) assemblages across a natural forest-cerrado ecotone in Minas Gerais, Brazil. Neotropical Entomology, v.34, n.5, p.721-731, 2005. Disponível em: <http://www.scielo.br/pdf/ne/v34n5/ a03v34n5.pdf $>$. Acesso em: 09 ago. 2012.

FLECHTMANN, C.A.H. et al. Controle biológico da mosca-doschifres (Haematobia irritans irritans) em Selvíria, Mato Grosso do Sul. 4. Comparação entre métodos de coleta de besouros coprófagos (Scarabaeidae). Revista Brasileira de Entomologia, v.39, n.2, p.259-276, 1995a. Disponível em: <http://www.agr.feis. unesp.br/cahf/home/H_Pub/Pub/fle_j009.pdf>. Acesso em: 15 ago. 2012.

FLECHTMANN, C.A.H. et al. Controle biológico da mosca-doschifres (Haematobia irritans irritans) em Selvíria, Mato Grosso do Sul. 5. Seleção de besouros coprófagos. Revista Brasileira de Entomologia, v.39, n.2, p.277-286, 1995b. Disponível em: <http://www.agr.feis.unesp.br/cahf/home/H_Pub/Pub/fle_j010. pdf>. Acesso em: 09 ago. 2012.

FLECHTMANN, C.A.H. et al. Controle biológico da mosca-doschifres (Haematobia irritans irritans) em Selvíria, Mato Grosso do Sul. 3. Levantamento de espécies fimícolas associadas à mosca. Revista Brasileira de Entomologia, v.39, n.2, p.249-258, 1995c. Disponível em: <http://www.agr.feis.unesp.br/cahf/home/H_Pub/ Pub/fle_j010.pdf>. Acesso em: 01 out. 2012.

FONSECA, V.M.O.; KERR, W.E. Avaliações de índices reprodutivos em besouro africano (Digitonthophagus gazella Fabricius) (Coleoptera: Scarabaeidae). Bioscience Journal, v.21, n.3, p.61-68, 2005. Disponível em: <http://www.seer.ufu.br/index. php/biosciencejournal/article/view/6608/4341>. Acesso em: 15 ago. 2012.

GENIER, F. A revision of the neotropical genus Ontherus Erichson (Coleoptera: Scarabaeidae, Scarabaeinae), Supplement 1. Coleopterists Bulletin, v.52, n.3, p.270-274, 1998.

GILLETT, C.P.D.T. et al. Diversity and distribution of the scarab beetle tribe Phanaeini in the northern states of the Brazilian Northeast (Coleoptera: Scarabaeidae: Scarabaeinae). Insecta Mundi, v.118, p.1-19, 2010. Disponível em: <http:// digitalcommons.unl.edu/insectamundi/642>. Acesso em: 15 ago. 2012.

GRIES, R. et al. Evaluating the impacts and conservation value of exotic and native tree afforestation in Cerrado grasslands using dung beetles. Insect Conservation and Diversity, v.5, p.175185, 2012. Disponível em: <http://onlinelibrary. wiley.com/ doi/10.1111/j.1752-4598.2011.00145.x/pdf>. Acesso em: 27 dez. 2012. doi: 10.1111/j.1752-4598.2011.00145.x.
GROSSI, P.C.; GROSSI, E.J. A new species of Brachysiderus Waterhouse, 1881 (Coleoptera: Scarabaeidae: Dynastinae) from Maranhão State, Brazil. Zootaxa, v.1054, p.25-29, 2005.

HOWDEN, H.F. New species and a new subgenus of South American Neoathyreus Howden and Martínez (Coleoptera: Geotrupidae: Athyreini). Zootaxa, v.1137, p.37-52, 2006.

JOLY, L.J.; ESCALONA, H. Revisión del género Chalepides Casey, 1915 (Coleoptera: Scarabaeidae: Dynastinae: Cyclocephalini). Entomotropica, v.17, n.1, p.37-90, 2002.

KOLLER, W.W. et al. Besouros coprófagos (Coleoptera: Scarabaeidae) coletados em Campo Grande, MS, Brasil. Anais da Sociedade Entomológica do Brasil, v.28, n.3, p.403-412, 1999.

KOLLER, W.W. et al. Scarabaeidae e Aphodiidae coprófagos em pastagens cultivadas em área do cerrado sul-mato-grossense. Revista Brasileira de Zoociências, v.9, n.1, p.81-93, 2007. Disponível em: <http://www.editoraufjf.com.br/revista/index.php/ zoociencias/article/viewFile/140/130>. Acesso em: 02 dez. 2012.

LOUZADA, J.N.C.; SILVA, P.R.C. Utilisation of introduced Brazilian pastures ecosystems by native dung beetles: diversity patterns and resource use. Insect Conservation and Diversity, v.2, n.1, p.45-52, 2009. Disponível em: <http://onlinelibrary. wiley.com/doi/10.1111/j.1752-4598.2008.00038.x/pdf>. Acesso em: 02 dez. 2012. doi: 10.1111/j.1752-4598.2008.00038.x.

MARCHIORI, C.H. et al. Artrópodos associados com carcaça de suíno em Itumbiara, sul de Goiás. Arquivos do Instituto Biológico de São Paulo, v.67, n.2, p.167-170, 2000a. Disponível em: <http:// www.biologico.sp.gov.br/docs/arq/V67_2/4.pdf>. Acesso em: 27 nov. 2012.

MARCHIORI, C.H. et al. Levantamento de Coleoptera (Arthropoda: Insecta) associados a fezes de gado bovino, no município de Itumbiara, Goiás, Brasil. Acta Scientiarum, v.22, n.2, p.403-407, 2000b. Disponível em: <http://eduemojs.uem.br/ojs/index.php/ ActaSciBiolSci/article/view/2888/2107>. Acesso em: 27 nov. 2012.

MARCHIORI, C.H. et al. Succession of Scarabaeidae on bovine dung in Itumbiara, Goiás, Brazil. Neotropical Entomology, v.32, n.1, p.173-176, 2003. Disponível em: <http://www.scielo.br/pdf/ne/ v32n1/15591.pdf>. Acesso em: 19 nov. 2012.

MARCHIORI, C.H. Insects (Arthropoda: Insecta) collected on bovine feces after different times of field exposure in Itumbiara, Goias, Brazil. Arquivos do Instituto Biológico, v.70, n.3, p.377-380, 2003.

MARTINS, E.; CONTEL, E.P.B. Dados biológicos da criação do besouro africano Onthophagus gazella Fabricius (Scarabaeidae) em terrários na fazenda Experimental Getúlio Vargas de Uberaba (MG). Revista Brasileira de Biologia, v.57, n.3, p.403-409, 1997.

MILHOMEM, M.S. et al. Técnicas de coleta de besouros copronecrófagos no Cerrado. Pesquisa Agropecuária Brasileira, v.38, n.11, p.1249-1256, 2003. Disponível em: <http://www. scielo.br/pdf/pab/v38n11/18918.pdf>. Acesso em: 13 set. 2012.

MITTERMEIER, R.A. et al. Hotspots revisited: Earth's biologically richest and most threatened terrestrial ecoregions. Washington, D.C.: Conservation International, 2005. 392p.

MORÓN, M.A. Diversidade y distribución del complejo "gallina ciega” (Coleoptera: Scarabaeoidea). In: RODRÍGUEZ DEL 
BOSQUE, L.A.; MORÓN, M.A. Plagas del suelo. México: Mundi-prensa México, 2010. Cap.3, p.41-64.

NICHOLS, E. et al. Ecological functions and ecosystem services provided by Scarabaeinae dung beetles. Biological Conservation, v.141, n.6, p.1461-1474, 2008. Disponível em: <http://www. sciencedirect.com/science/article/pii/S0006320708001420\#>. Acesso em: 13 set. 2012.

NUNES, R.V.; FRIZZAS, M.R. Microbiota da flora intestinal de besouros (Coleoptera: Scarabaeidae) no Parque Olhos d'Água. Universitas: Ciências da Saúde, v.5, n.1/2, p.1-13, 2007. Disponível em: <http://www.publicacoes.uniceub.br/index.php/ cienciasaude/article/viewArticle/429>. Acesso em: 27 nov. 2012. doi: 10.5102/ucs.v5i1.429

NUNES, R.V. et al. Scarabaeinae (Coleoptera: Scarabaeidae) of a rupestrian field at Cafuringa, Distrito Federal, Brazil: commented list of species. Biota Neotropica, v.12, n.4, p.15, 2012. Disponível em: <http://dx.doi.org/10.1590/S167606032012000400013>. Acesso em: 28 jun. 2012. doi: 10.1590/ S1676-06032012000400013.

OCAMPO, F.C. A new species of the Neotropical genus Daimothoracodes Petrovitz (Coleoptera: Scarabaeoidea: Hybosoridae). Zootaxa, v.1048, p.45-51, 2005. Disponível em: <http://216.92.149.63/zootaxa/2005f/z01048p051f.pdf>. Acesso em: 15 jul. 2012.

OCAMPO, F.C.; VAZ-DE-MELLO, F.Z. A new species of Daimothoracodes Petrovitz (Coleoptera: Scarabaeoidea: Hybosoridae) and a key to species of the genus. Proceedings of the Entomological Society of Washington, v.104, n.4, p.912-917, 2002.

OLIVEIRA, C.M. et al. Aegopsis bolboceridus (Coleoptera: Melolonthidae): an important pest on vegetables and corn in Central Brazil. Florida Entomologist, v.91, n.2, p.324-327, 2008. Disponível em: <http:/www.bioone.org/doi/pdf/10.1653/00154040\%282008\%2991\%5B324\%3AABCMAI\%5D2.0.CO\%3B2>. Acesso em: 25 set. 2012.

OLIVEIRA, C.M. et al. First record of Phyllophaga sp aff. capillata (Coleoptera: Melolonthidae) as a soybean pest in the Brazilian "Cerrado". Florida Entomologist, v.90, n.4, p.772-775, 2007. Disponível em: <http://www.bioone.org/doi/ pdf/10.1653/0015-4040\%282007\%2990\%5B772\%3AFROPSA\% 5D2.0.CO\%3B2>. Acesso em: 25 set. 2012.

OLIVEIRA, H.N.; ÁVILA, C.J. Ocorrência de Cyclocephala forsteri em Acronomia aculeata. Pesquisa Agropecuária Tropical, v.41, n.2, p.293-295, 2011. Disponível em: <http:// www.revistas.ufg.br/index.php/pat/article/view/8769>. Acesso em: 15 dez. 2012.

PUKER, A. et al. Espécies de Scarabaeidae fitófagos (Insecta: Coleoptera) associadas ao sistema radicular de Acrocomia aculeata (Jacq.) Lodd. ex Mart. (Arecaceae). Biota Neotropica, v.9, n.3, p.105-109, 2009. Disponível em: <http://www.scielo.br/ pdf/bn/v9n3/v9n3a09.pdf>. Acesso em: 16 nov. 2012.

PUKER,A. et al. First record of Leucothyreus albopilosus (Coleoptera: Scarabaeidae: Rutelinae: Geniatini) in lesions on stem of Eucalyptus citriodora and beehives of Apis mellifera. Entomological Science, v.14, p.230-233, 2011b. Disponível em: <http://onlinelibrary.wiley. com/doi/10.1111/j.1479-8298.2010.00433.x/pdf>. Acesso em: 11 nov. 2012. doi: 10.1111/j.1479-8298.2010.00433.x.
PUKER, A. et al. Notes on biology and behavior of rhinoceros beetle Enema pan (Coleoptera: Scarabaeidae: Dynastinae). Annals of the Entomological Society of America, v.104, n.5, p.919-927, 2011a. Disponível em: <http://www.bioone.org/doi/ pdf/10.1603/AN10197>. Acesso em: 15 jul. 2012.

RESENDE, N.F. Mudanças do uso da terra no Cerrado sobre comunidade de besouros escarabeíneos (Coleoptera: Scarabaeidae: Scarabaeinae). Revista Brasileira de Gestão e Engenharia, v.5, p.87-102, 2012. Disponível em: <http://www.periodicos.cesg.edu. br/index.php/gestaoeengenharia/article/view/73/97>. Acesso em: 27 dez. 2012

RODRIGUES, S.R. et al. Aspectos biológicos de Cyclocephala verticalis Burmeister (Coleoptera: Scarabaeidae). Neotropical Entomology, v.39, n.1, p.15-18, 2010a. Disponível em: <http:// www.scielo.br/pdf/ne/v39n1/v39n1a03.pdf>. Acesso em: 27 ago. 2012.

RODRIGUES, S.R. et al. Aspectos biológicos de Leucothyreus dorsalis Blanchard (Coleoptera, Scarabaeidae, Rutelinae). Revista Brasileira de Entomologia, v.54, n.3, p.431-435, 2010b. Disponível em: <http://dx.doi.org/10.1590/S008556262010000300012>. Acesso em: 27 ago. 2012. doi: 10.1590/ S0085-56262010000300012.

RODRIGUES, S.R. et al. Occurrence of adults and biological aspects of Geniates borelli Camerano (Coleoptera, Scarabaeidae, Rutelinae) in Aquidauana, MS, Brazil. Revista Brasileira de Entomologia, v.56, n.3, p.315-318, 2012. Disponível em: <http://www.scielo.br/pdf/ rbent/v56n3/aop3512.pdf>. Acesso em: 27 dez. 2012.

RODRIGUES, S.R. et al. Occurrence, biology and behavior of Liogenys fuscus Blanchard (Insecta, Coleoptera, Scarabaeidae) in Aquidauana, Mato Grosso do Sul, Brazil. Revista Brasileira de Entomologia, v.52, n.4, p.637-640, 2008b. Disponível em: $<$ http://www.scielo.br/pdf/rbent/v52n4/a15v52n4.pdf>. Acesso em: 27 ago. 2012.

RODRIGUES, S.R. et al. Ocorrência de larvas de Scarabaeidae fitófagos (Insecta: Coleoptera) em diferentes sistemas de sucessão de culturas. Pesquisa Agropecuária Tropical, v.41, n.1, p.87-93, 2011. Disponível em: <http://www.revistas.ufg.br/index.php/pat/ article/view/7698>. Acesso em: 27 ago. 2012.

RODRIGUES, S.R. et al. Ocorrência e aspectos biológicos de Anomala testaceipennis Blanchard (Coleoptera, Scarabaeidae). Revista Brasileira de Entomologia, v.52, n.1, p.68-71, 2008a. Disponível em: <http://www.scielo.br/pdf/rbent/v52n1/12.pdf>. Acesso em: 27 ago. 2012.

RODRIGUES, S.R.; FALCO, J.S. Aspectos biológicos de Pelidnota fulva Blanchard, 1850 (Coleoptera, Scarabaeidae, Rutelinae). Biota Neotropica, v.11, n.1, p.157-160, 2011. Disponível em: <http://www.scielo.br/pdf/bn/v11n1/15.pdf>. Acesso em: 27 ago. 2012.

RODRIGUES, S.R.; FLECHTMANN, C.A.H. Aspectos biológicos de Canthon lituratus (Germar, 1813) e Canthidium (Canthidium) megathopoides Boucomont, 1928 (Coleoptera, Scarabaeidae). Acta Zoológica Mexicana, v.70, p.1-12, 1997. Disponível em: <http://www.agr.feis.unesp.br/cahf/home/H_Pub/ Pub/fle_j018.pdf>. Acesso em: 28 ago. 2012.

ROSA, T.A. et al. Arthropods associated with pig carrion in two vegetation profiles of Cerrado in the State of Minas Gerais, Brazil. 
Revista Brasileira de Entomologia, v.55, n.3, p.424-434, 2011. Disponível em: <http://www.scielo.br/pdf/rbent/v55n3/aop4511. pdf $>$. Acesso em: 27 nov. 2012.

SANTOS, A.C. et al. Chemical control of white grub Liogenys fuscus (Blanchard 1851) (Coleoptera: Melolonthidae) in cornfields. BioAssay, v. 3, p.1-6, 2008. Disponível em: <http:// www.bioassay.org.br/bioassay/article/download/59/91>. Acesso em: 28 maio, 2013

SANTOS, V.; ÁVILA, C.J. Aspectos bioecológicos de Cyclocephada forsteri Endrodi, 1963 (Coleoptera: Melolonthidae) no Estado do Mato Grosso do Sul. Revista de Agricultura, v.82, n.1, p.28-30, 2007.

SANTOS, V.; ÁVILA, C.J. Aspectos biológicos e comportamentais de Liogenys suturalis Blanchard (Coleoptera: Melolonthidae) no Mato Grosso do Sul. Neotropical Entomology, v.38, n.6, p.734-740, 2009a. Disponível em: <http://www.scielo.br/pdf/ne/ v38n6/05.pdf>. Acesso em: 01 ago. 2012

SANTOS, V.; AVILA, C.J. Ocorrência de parasitismo em larvas de Liogenys suturalis Blanchard, 1851 (Coleoptera: Melolonthidae) por Ptilodexia Brauer \& Bergenstamm, 1889 (Diptera: Tachinidae). Arquivos do Instituto Biológico, v.76, n.4, p.745-748. 2009b. Disponível em: <http://www.biologico.sp.gov.br/docs/arq/v76_4/ santos2.pdf>. Acesso em: 28 maio, 2013.

SILVA, R.J. et al. Heterogeneidade do habitat, riqueza e estrutura da assembléia de besouros rola-bostas (Scarabaeidae: Scarabaeinae) em áreas de Cerrado na Chapada dos Parecis, MT. Neotropical Entomology, v.39, n.6, p.934-940, 2010. Disponível em: <http://www.scielo.br/pdf/ne/v39n6/v39n6a14.pdf >. Acesso em: 02 dez. 2012.

SILVEIRA, F.A.O. et al. Predation on Atta Iaevigata (Smith 1858) (Formicidae Attini) by Canthon virens (Mannerheim 1829)
(Coleoptera Scarabaeidae). Tropical Zoology, v.19, p.1-7, 2006. Disponível em: <http://91.121.146.53/index.php/tropicalzoology/ article/download/127/125>. Acesso em: 18 out. 2012.

STEBNICKA, Z.T. A second species of Selviria Stebnicka from Brazil (Coleoptera: Scarabaeidae: Eupariini). Coleopterists Bulletin, v.59, n.1, p.23-25, 2005. Disponível em: <http://www. bioone.org/doi/pdf/10.1649/678>. Acesso em: 15 ago. 2012.

STEBNICKA, Z.T. Neotropical Eupariini: new and little known genera and species (Coleoptera: Scarabaeoidea: Aphodiinae) . Revue Suisse de Zoologie, v. 106, n.2, p.285300, 1999.

UCHOA, T.; MOURA-BRITTO, M. Hábito alimentar e uso do habitat por canídeos no Parque Estadual do Cerrado: avaliação da situação atual da família Canidae no limite sul do bioma Cerrado no Brasil. Cadernos da biodiversidade, v.4, n.2, p.59-65, 2004. Disponível em: <http://ag20.cnptia.embrapa.br/Repositorio/ Canideos_Sul_CerradoID-f7X9KrdMSX.pdf>. Acesso em: 20 dez. 2012

VAZ-DE-MELLO, F.Z.; CANHEDO, V.L. Duas novas espécies brasileiras de Pedaridium (Coeloptera, Scarabaeidae, Coprinae, Ateuchini). Iheringia, Serie Zoologia, v.84, p.95-100, 1998

VAZ-DE-MELLO, F.Z.; GÉNIER, F. Notes on the behavior of Dendropaemon Perty and Tetramereia Klages (Scarabaeidae: Scarabaeinae: Phanaeini). Coleopterists Bulletin, v.63, n.3, p.364-366, 2009. Disponível em: <http://www.bioone.org/doi/ pdf/10.1649/1191.1>. Acesso em: 05 dez. 2012.

VAZ-DE-MELLO, F.Z.; GROSSI, P.C. First report of Bolbites Harold (Scarabaeidae: Scarabaeinae: Phanaeini) in Brazil. Coleopterists Bulletin, v.64, n.3, p.220-220, 2010. Disponível em: <http://www.bioone.org/doi/pdf/10.1649/0010-065X-64.3.220.6>. Acesso em: 11 set. 2012. 